\title{
L'École en France de 1945 à nos jours, André D. Robert
}

Grenoble, Presses universitaires de Grenoble, 2010, 312 p.

\section{Roger-François Gauthier}

\section{CpenEdition}

\section{Journals}

Édition électronique

URL : https://journals.openedition.org/ries/1029

DOI : $10.4000 /$ ries. 1029

ISSN : 2261-4265

Éditeur

France Education international

Édition imprimée

Date de publication : 1 avril 2011

Pagination : 24-25

ISBN : 978-2854205916

ISSN : 1254-4590

Référence électronique

Roger-François Gauthier, "L'École en France de 1945 à nos jours, André D. Robert », Revue internationale d'éducation de Sèvres [En ligne], 56 | avril 2011, mis en ligne le 01 avril 2011, consulté le 04 mai 2021. URL : http://journals.openedition.org/ries/1029 ; DOI : https://doi.org/10.4000/ries.1029

Ce document a été généré automatiquement le 4 mai 2021.

(c) Tous droits réservés 


\section{L'École en France de 1945 à nos jours, André D. Robert}

Grenoble, Presses universitaires de Grenoble, 2010, 312 p.

Roger-François Gauthier

\section{RÉFÉRENCE}

L'École en France de 1945 à nos jours, André D. Robert, Grenoble, Presses universitaires de Grenoble, 2010, 312 p.

1 Ce nouvel ouvrage d'André Robert, notamment directeur du laboratoire Éducation, Cultures \& Politiques (Lyon 2), fournit une histoire aussi complète qu'accessible de l'éducation en France sur soixante ans, d'une façon qui conjugue deux exigences :

- proposer pragmatiquement une histoire des « politiques scolaires [françaises] au jour le jour » (p. 7), pour insister sur la « dimension factuelle du récit », mais dans le cadre d'un séquençage qui permet au lecteur d'arriver à une mise en ordre qui permet la compréhension ;

- inviter le lecteur à réfléchir de façon distanciée à la question de l'«inflexion de la société française et de son école » (p. 15) au cours de la longue période envisagée.

2 Si le risque couru par tous les ouvrages d'histoire de l'éducation qui portent sur la résurrection de périodes entières est en effet celui d'un discours qui, se voulant objectif, ne parvient qu'à être descriptif et plat, André Robert prend les devants en avertissant son lecteur qu'il écrit à partir d'un point explicite de pensée et d'écriture (p. 17) : «... nos choix personnels nous inclinent vers un type de société démocratique », société notamment définie par l'existence de «dépenses budgétaires tournées vers l'intérêt général, notamment en matière d'éducation...». Cette prise de risque de l'auteur rend cette lecture particulièrement intéressante, car, en permanence, comme dans un "pianotement à côté " (Stéphane Mallarmé), il s'interroge sur ce qui s'est passé pour que des idées et des positionnements, notamment se rattachant à une critique anti-capitaliste, qui semblaient hier incontournables, aient visiblement été 
aujourd'hui remisés, parfois sous la plume des mêmes théoriciens, «au placard des idées mortes » (p. 5).

Le séquençage commence avec la préparation du plan Langevin-Wallon (1947), c'est-àdire avec l'élaboration de la référence mythique de la pensée "compréhensive " sur l'école en France, pour aller jusqu'à presque nos jours (on peut regretter, même si la crainte de la myopie pour la contemporanéité retient légitimement l'historien, que l'action éducative des premières années de la présidence Sarkozy ne soit pas étudiée).

Le paysage de départ est bien sûr celui de l'État éducateur à la française, où l'éducation est chargée de "pacifie[r] la société et d'assure[r] (...) l'ordre nécessaire à la prospérité économique de la bourgeoisie » (B. Charlot) : c'est le temps de l'« école séparatrice » (p. 11) entre le peuple et les classes dirigeantes.

5 Au sortir de la Seconde Guerre mondiale, c'est «l'école conquérante », avec pendant une trentaine d'années une sorte $d^{\prime}$ '« alliance » (V. Troger) objective entre économistes et "partisans de l'école nouvelle» (id.), même si des travaux aussi forts que La reproduction de P. Bourdieu et J.-C.Passeron (1970) contestent dès l'époque cette confiance. Cette période est toutefois complexe, si on analyse l'écart entre les «rêves réformateurs» et la non-décision (cela jusqu'à 1958 et l'arrivée de la Cinquième République), puis les grandes décisions prises par le général de Gaulle afin d'«assurer une prospection aussi complète que possible de nos ressources juvéniles». Le chapitre 3, qui se rattache à cette période, propose un examen détaillé de la question de «1968, ses enjeux et conséquences dans l'Éducation nationale (1968-1972)», en essayant d'intégrer cette période étonnante dans les évolutions à plus longue échelle, en montrant que ce que les ambiguités de ce mouvement purent annoncer des ambiguïtés des débats scolaires ultérieurs.

6 Avec les effets de la première crise pétrolière, l'école devient « hésitante ", et Robert montre que ces hésitations caractérisèrent aussi bien les gouvernements de droite - qui certes créent le "collège unique ", mais avec des aléas de réception et de mise en œuvre qui permettent de parler de «nébuleuse » de la réforme Haby (1973-1980) - que ceux de la gauche arrivée au pouvoir en 1981 : d'abord, avec Alain Savary, la gauche se situe dans un cadre qu'on peut initialement croire consensuel, avant de trébucher en 1984 sur le contentieux public/privé ; Jean-Pierre Chevènement arrive rue de Grenelle en affichant d'une part ses conceptions scolaires républicaines et traditionnalistes, en déployant d'autre part une politique modernisatrice (objectif quantitatif des $80 \%$ d'une classe d'âge au baccalauréat) de rapprochement systématique école/entreprise (création des baccalauréats professionnels, par exemple).

7 Trouble à gauche comme à droite, c'est la situation de 1986, que le rapport remis en 1985 au président de la République par le Collège de France, explicite en montrant, selon Derouet, que, désormais, l'école est entrée dans un univers à justification multiple (intérêt général, efficacité, principe marchand, faveur de la créativité, etc.).

Les années suivantes permettent selon l'auteur aussi bien de voir, jusqu'en 2002, les valses-hésitations des gouvernants, de droite comme de gauche, que, depuis, de poser la question (chap.9) de savoir si l'éducation nationale a changé ses concepts de référence, en étant «durablement saisie par le néolibéralisme ». Les enjeux des deux grandes lois de 1989 et de 2005 sont analysés avec précision, ce qui est particulièrement précieux. 
9 Le dernier adjectif à accoler à l'école française est-il celui de "postmoderne ", comme s'il était admis que «l'ancien principe de l'acquisition du savoir... indissociable de la formation de l'esprit [...] tombe [...] en désuétude » (Jean-François Lyotard) ? Robert ne le pense pas, car il considère que toutes les idéologies agissantes en France en matière scolaire "visent encore à conférer une direction et une certaine densité à l'école (p. 262) ». Le chapitre de conclusion réfléchit à la notion de "politique éducative » à partir de cette observation.

Petite critique face à un ouvrage qui va devenir référence pour tous ceux qui veulent comprendre les évolutions de l'école en France : on aurait pu souhaiter que l'on sache mieux non pas dans quelle mesure des influences étrangères ont pesé sur l'histoire de l'école en France entre 1945 et 2005, car cela est souvent expliqué, mais dans quelle mesure cette histoire est spécifique. Le système français a-t-il marqué plus ou moins de résistance au changement que les autres? Que peut-on en penser? Moins pour interroger le passé, d'ailleurs, que pour interroger le futur et savoir quel sera le prochain chapitre.

\section{AUTEURS}

\section{ROGER-FRANÇOIS GAUTHIER}

Roger-François Gauthier est inspecteur général de l'administration de l'Education nationale et de la recherche où il a travaillé notamment sur les questions des acquis des élèves et des étudiants et de leurs évaluations. Docteur en sciences de l'éducation (Université de Lyon 2), il est par ailleurs consultant auprès de l'Unesco, où il a publié en français et en anglais un rapport sur les contenus d'enseignement dans le secondaire dans le monde. Auteur de nombreux articles et ouvrages en matière d'éducation, associé à plusieurs équipes de recherche, il est également viceprésident de l'Association française des administrateurs de l'éducation (AFAE), membre de la Standing International Conference of Inspectorates (SICI), professeur associé à l'Université de Paris 5 La Sorbonne (politique éducative), et membre du comité de rédaction de la RIES. 